\title{
Some aspects of the behavior of metastable austenitic steels at high strain rates
}

\author{
Matti Isakov ${ }^{1 *}$, Lalit Pun ${ }^{1}$, Veli-Tapani Kuokkala ${ }^{1}$ and Mikko Hokka ${ }^{1}$ \\ ${ }^{1}$ Tampere University, Faculty of Engineering and Natural Sciences, Engineering Materials Science, \\ POB 589, FI-33014, Tampere, Finland
}

\begin{abstract}
Metastable austenite containing steels, i.e., steels capable of undergoing solid-state phase transformation from austenite to $\alpha^{\prime}$-martensite during plastic deformation, offer a very good combination of ductility, strength, and above all, exceptional strain hardening capability. In effect, in suitable plastic deformation conditions the relatively soft austenitic phase can transform to the harder $\alpha^{\prime}$-martensite, which increases the strain hardening rate of the material through various mechanisms. This special feature gives these kinds of alloys several beneficial properties, such as resistance against flow instabilities and increased capability to absorb deformation energy. For this reason, metastable austenite containing alloys have been extensively studied in the past. However, several open questions still remain, especially in the field of high rate deformation. This can be related to the great number and complexity of the related microstructural phenomena and their combined effects on the material response. The open questions affect both the metallurgy of the material and the numerical modeling of material behavior. The current contribution addresses some of these questions and their possible solutions, as well as gives an outlook on the possible future development directions.
\end{abstract}

\section{Introduction}

Metastable austenite containing steels have been studied, developed, and used for decades. The attention, which this group of materials has received, is well justified. In general, the metastable nature of austenite in steels and the resulting phase transformation to hard $\alpha$ 'martensite brings about excellent means to control the mechanical properties of the material via heat treatments. In the case of so-called strain-induced $\alpha^{\prime}$-transformation, in which the phase transformation takes place during plastic deformation, there exists a great potential to develop materials with well controlled strength, ductility, and strain hardening properties. In the field of high rate loading, the above mentioned properties are often related to the energy absorption capability of the material.

Despite the years of research, the topic continues to receive interest, as new alloys, such as those based on the Quench and Partitioning treatment are developed and as the knowledge on older alloys is deepened. In the case of high rate loading, one major challenge seems to persist: in general, the tendency towards strain-induced $\alpha^{\prime}$-transformation decreases as the

*Corresponding author: matti.isakov@tuni.fi 
rate of deformation increases. This observation is old and has been repeated numerous times in the field. One could even say that it has been almost accepted as an unsurmountable matter of fact. However, if one could somehow make the phase transformation persist at high rates of deformation, then one would be rewarded with a material that has outstanding strength and ductility properties on a wide range of loading rates. Therefore, in the authors' opinion, the topic deserves further attention.

It is suggested here that the research methodology concerning the strain rate dependent behavior of metastable austenite containing alloys should be somewhat reoriented. As will be shown below, it seems that the conventionally used approaches do not reveal all the aspects of the strain rate dependent plasticity of metastable austenite. Thus, both the use of existing alloys and the development of new ones, as well as material model development, might be unnecessarily limited due to insufficient knowledge of the underlying phenomena.

\section{On the limitations of constant strain rate tests}

Usually the strain rate dependent response of metastable austenite containing steels is measured, analyzed and modeled based on conventional stress-strain data, i.e., based on a test series where the strain rate (and sometimes temperature) is varied between the tests in the series but kept constant during any individual tests. This approach is in general sound and well-established in materials science and mechanical engineering: the underlying premise is that other variables (such as temperature) are kept constant while one variable (here strain rate) is systematically changed.

In the case of metastable austenite containing steels the above mentioned approach can be subjected to criticism, which is explained by referring to Fig. 1a: a mechanical test (e.g. uniaxial tension or compression) carried out at a sufficiently low strain rate allows to maintain constant strain rate and temperature, whereas plastic strain and material microstructure (such as volume fraction of strain induced $\alpha^{\prime}$-martensite) change during the test. In a similar test carried out at a higher strain rate the same reasoning applies, except that the material temperature tends to increase during the test due to the conversion of plastic deformation work into heat under the conditions of insufficient heat transfer.

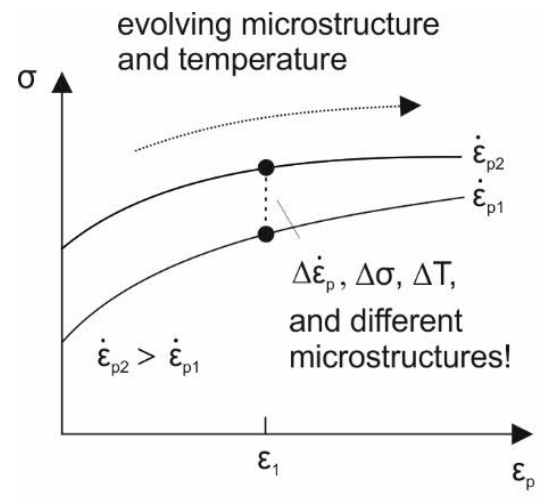

a)

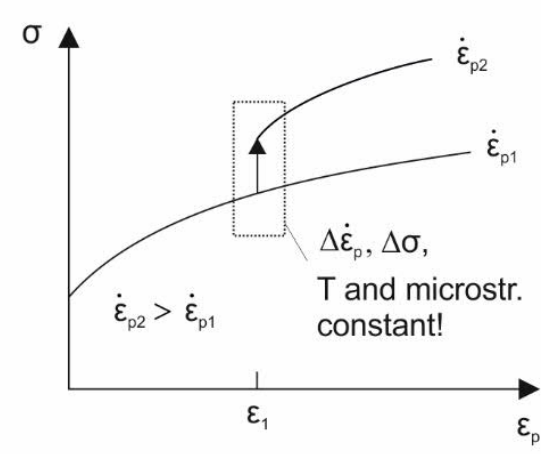

b)

Fig. 1. Schematic illustration of the different approaches used to study the strain rate effects in materials: a) evaluation based on comparing tests carried out at different strain rates involves a comparison between different microstructures and material temperatures, $b$ ) sudden change in strain rate (jump) reveals the effects of strain rate on flow stress and strain hardening rate in constant microstructure with small or negligible change in temperature. 
The statement regarding the microstructural evolution appears almost trivial at the first glance but becomes crucial when one considers the way the test data obtained at different strain rates is interpreted (Fig. 1a). Typically, a certain value (e.g., $10 \%$ ) is selected for the plastic strain and the corresponding flow stress values are read from the stress-strain curves corresponding to various strain rates (with strain rate being constant in an individual test). The possible differences in the flow stress are then interpreted as the "strain rate effect". The main question raised here is: an effect respect to what? Except for the yield strength (zero plastic strain), the flow stress values read from the curves of different tests do not correspond to the same microstructure nor temperature. For example, the data published by VázquezFernández [1] for metastable austenitic stainless steel EN 1.4318 shows that in the specimens deformed at different strain rates a 40 percentage point difference in the $\alpha^{\prime}$-martensite volume fraction and over $40 \mathrm{~K}$ difference in the material temperature can develop towards the end of the test. It is evident that in such case it must be carefully considered how the differences in the flow curves measured at different strain rates are interpreted.

The discussion presented above points out that the approaches used to study the strain rate effects in metastable austenite containing steels should be critically evaluated. A general approach, which seems to offer a robust base for the analysis as well as for material model development, can be briefly summarized in the equation form as:

$$
\begin{aligned}
& \sigma=f\left(\text { microstr }, T, \dot{\varepsilon}_{p}\right) \\
& \frac{d(\text { microstr })}{d \varepsilon}=g\left(T, \dot{\varepsilon}_{p}\right)
\end{aligned}
$$

As is evident in Equation 1, strain is not used to describe the flow stress of the material. The reasoning behind this is that strain cannot be considered as a true state variable, since it does not take into account the full history of deformation. As discussed above, two test specimens might have undergone exactly the same amount of strain prior to the point of evaluation but have a completely different microstructural state due to the differences in their loading history (such as strain rate and/or temperature). Strain rate, in contrast, can be considered as a state variable, since it is a measure of the rate at which the microstructure is currently deforming. It should also be noted that even though Equation 1 explicitly includes strain rate and temperature, this alone is not enough to describe the thermomechanical response of the material since, as shown in Equation 2, also the microstructural evolution can be a function of strain rate and temperature, as noted above.

The above presented argument has been made several times in the literature in conjunction with various materials, and the authors believe that it is very important also in the case of metastable austenitic alloys, in which extensive microstructural changes take place during plastic deformation. In fact, the above discussed "dilemma" of different microstructures in constant strain rate tests disappears, when one discards strain as the "leading" variable in the analysis. Furthermore, in this approach constant strain rate experiments are only one means to study material behavior, i.e., they are mainly used to study the effects of deformation conditions on microstructure evolution, not to evaluate the properties of a certain microstructure. For the latter purpose, the so-called jump tests are more feasible. For example, in a strain rate jump test the imposed strain rate is suddenly changed during the test and the resulting changes in the flow stress and the slope of the stress-strain curve are measured (Fig. 1b). This method gives direct information on the strain rate sensitivity of a certain microstructure with negligible or only small change in the material temperature during the jump.

A review on the literature shows that, although not being numerous, reports on strain rate jump tests for metastable austenite containing steels have been intermittently published over the last decades [1-7]. These studies vary in their focus. In some works, strain rate jumps 
were used to determine the strain rate sensitivity index [2-4], whereas for example Callahan et al. [6] used the jump tests to analyze the interaction between dynamic strain aging and $\alpha^{\prime}-$ martensite transformation. In another group of works, the effects of strain rate and adiabatic heating on the phase transformation kinetics were analyzed using strain rate jump tests $[1,5$, 7]. The high strain rate tests on prestrained material reported by Larour et al. [8] can also be counted in this group. These works reveal an interesting but so far relatively little studied aspect of the strain-induced $\alpha^{\prime}$-martensite transformation: it appears that in addition to adiabatic heating, which is the common explanation for the increased austenite stability at high strain rates, also the strain rate itself seems to play an important role in the transformation kinetics. This finding will be discussed below in more detail.

\section{Direct strain rate effects on the $\alpha$-transformation}

The possible direct effects of strain rate on the strain-induced $\alpha^{\prime}$-phase transformation were discussed already in the late 1970s $[9,10]$. According to these studies, high strain rate may to some extent promote the transformation, since the resulting higher flow stress can increase the number of nucleation sites such as the intersection volumes of shear bands, which are themselves composed of overlapping stacking faults. This effect is, however, limited to small strains before adiabatic heating starts to suppress the phase transformation. Later, Talonen [11] concluded that the above mentioned effect takes place to some (minor) extent in a relatively stable stainless steel alloy (EN 1.4301) but is absent in a readily transforming alloy (EN 1.4318). Furthermore, Hokka [12] found no evidence of a positive strain rate effect in a low alloyed TRIP steel; in fact, according to his results the phase transformation is suppressed at high strain rates already at low strains when the amount of bulk adiabatic heating should be low.

However, as noted above, during the recent years experimental evidence has been mounting that strain rate has a direct and notable effect on the $\alpha^{\prime}$-phase transformation and the strain hardening rate also at high strains. This evidence can be briefly summarized as follows: 1) Isakov et al. [5] observed that when the strain rate is instantaneously increased from the low strain rate isothermal conditions to the high strain rate adiabatic conditions, both the strain hardening rate and the $\alpha^{\prime}$-phase transformation rate decrease rapidly. Later, Vázquez-Fernández [1] repeated the mechanical tests with in-situ IR-measurements and showed that macroscopic adiabatic heating following the strain rate jump is modest and appears to be too low to cause the observed immediate decrease in the $\alpha^{\prime}$-phase transformation rate. Furthermore, recent independent measurements by Sunil and Kapoor [7] verify the instantaneous effect of strain rate change on the strain hardening rate, and a similar conclusion can be drawn from the data by Larour et al. [8], i.e., the strain hardening rate during a high rate test is more or less independent of the preceding prestraining. 2) If a high strain rate adiabatic test is interrupted and the specimen is reloaded in low strain rate isothermal conditions, the phase transformation proceeds readily without apparent "incubation strain", i.e., the transformation conditions are otherwise suitable during high rate deformation, but for some (yet unknown) reason the phase transformation does not proceed [5]. 3) The strain hardening and $\alpha^{\prime}$-phase transformation rates observed in a high rate adiabatic test cannot be replicated in a low rate test by applying external heating to mimic the macroscopic adiabatic temperature increase [13]. 4) Similarly, incremental loading at a high rate, which allows the specimen to cool down between the loadings, does not lead to the same phase transformation tendency as observed in low rate loading [7].

The evidence listed above may not be conclusive, but it gives reasonable grounds to suspect that the commonly presented theory, which explains the suppression of the $\alpha^{\prime}-$ transformation with bulk adiabatic heating, might be insufficient. This notion is in the authors' opinion extremely important; if the underlying mechanisms are better understood, 
perhaps the negative effects of high rate loading on the phase transformation tendency can be alleviated.

So far, the overall picture is still unclear. However, at least two (competing) hypotheses can be formulated. The first one considers microscale heating in lieu of bulk adiabatic heating (Fig. 2), i.e., the local slip activity near the newly formed $\alpha^{\prime}$-embryos and the heat release from the phase transformation might lead to the formation of "hot spots" in the microstructure, and thus the local temperature during high rate deformation might be considerably higher than the bulk macroscopic temperature. This idea was put forth by Talonen [11], and it is tempting since it is in agreement with the known growth mechanism of the $\alpha^{\prime}$-martensite, i.e., repeated nucleation and coalescence [10], and it can at least qualitatively explain many of the observations listed above. The rapid and notable decrease in the strain hardening and phase transformation rates following a sudden strain rate increase could happen because the $\alpha^{\prime}$-transformation is suddenly inhibited in the preferential microstructural locations, which quickly heat up after the strain rate is increased. Similarly, the "mismatch" observed between the high strain rate tests and the low strain rate tests with external heating [13] might take place because the external heating cannot reproduce the nonhomogeneous heating at the microstructural level. Similar reasoning applies to the incremental tests carried out by Sunil and Kapoor [7]; loading of the material incrementally only helps to cool down the material between the increments, but within each high rate increment the loading conditions are still adiabatic and local hot spots might form in the microstructure. At the moment, however, this theory lacks experimental evidence and thus remains only speculative. However, recent numerical simulations [14] have indicated that such hot spots are indeed plausible. This theory, if proven correct, might open up possibilities to mitigate the adiabatic heating effects on the phase transformation via tailoring of the microstructural morphology.

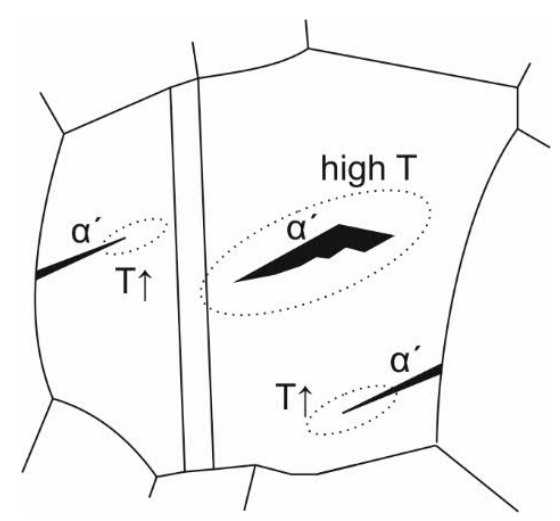

Fig. 2. Schematic illustration of the hypothesized inhibition mechanism of $\alpha^{\prime}$-transformation at high strain rates: increased slip activity and heat release from the phase transformation increase the local temperature $(\mathrm{T})$ in the microstructure and suppress further nucleation and coalescence of martensite embryos.

The hypothesis presented above can be challenged by an alternative hypothesis, i.e., that there exists a direct strain rate effect besides adiabatic heating. In fact, Sunil and Kapoor [7] hypothesized that reduced thermal activation at high strain rates decreases the stacking fault width, thus decreasing the number of nucleation points for the $\alpha^{\prime}$-martensite. However, this theory might be too simplistic and it also lacks experimental evidence: as noted above, high strain rates have been observed to rather promote than suppress the formation of shear bands, which are composed of stacking faults [9-11]. A direct strain rate effect might, though, take place via other means. In [5] it was shown that the strain rate dependency of the 
$\alpha^{\prime}$-transformation rate can be phenomenologically modeled with the well-known OlsonCohen model [9] with a combination of adiabatic temperature increase and strain rate dependent nucleation probability at the shear band intersections. This finding also requires more experimental evidence, but it sheds some light on the possible mechanism: perhaps the strain-induced $\alpha^{\prime}$-phase transformation involves a thermally activated part, which is related to the dislocation motion taking place in conjunction with the formation of the $\alpha^{\prime}$-martensite embryos?

\section{Conclusions}

To summarize, in this paper some of the key challenges related to the high strain rate deformation of metastable austenite containing steels were addressed. Firstly, the choice of the test methodology affects both the metallurgical analysis and the material model development. The conventionally used approach of comparing monotonous tests carried out at different strain rates does not reveal all the important features of the strain rate dependency of metastable austenite containing steels. It is argued here that such test data should be supplemented with tests involving changes of strain rate within one test in order to reveal all the effects of strain rate on these materials. Secondly, the recently acquired experimental evidence indicates that the kinetics of the strain-induced $\alpha^{\prime}$-martensite transformation during high strain rate deformation are not fully understood, i.e., the common theory based on bulk adiabatic heating appears insufficient in explaining all the experimental observations. Some alternative hypotheses reviewed in this paper highlight the necessity to continue the work on this topic.

\section{References}

1. N.I. Vázquez-Fernández, Doctoral Dissertation, Tampere University, Finland (2020)

2. G.L. Huang, D.K. Matlock, G. Krauss, Met. Trans. A 20A, 1239-1246 (1989)

3. V. Talyan, R.H. Wagoner, J.K. Lee, Met. Mat. Trans. A 29A, 2161-2172 (1998)

4. A. Kanni Raj, J. Satish Kumar, K.A. Padmanabhan, Mater. Lett. 38, 386-390 (1999)

5. M. Isakov, S. Hiermaier, V.-T. Kuokkala, Met. Mat. Trans. A 46A, 2352-2355 (2015)

6. M. Callahan, A. Perlade, J.-H. Schmitt, Mat. Sci. Eng. A 754, 140-151 (2019)

7. S. Sunil, R. Kapoor, Met. Mat.Trans. A 51A, 5667-5676 (2020)

8. P. Larour, P. Verleysen, K. Dahmen, W. Bleck, Steel Res. Int. 84, 72-88 (2012)

9. G.B. Olson, M. Cohen, Met. Trans. A 6A, 791-795 (1975)

10. L.E. Murr, K. P. Staudhammer, S.S. Hecker, Met. Trans. A 13A, 627-635 (1982)

11. J. Talonen, Doctoral Dissertation, Helsinki University of Technology, Finland (2007)

12. M. Hokka, Doctoral Dissertation, Tampere University of Technology, Finland (2008)

13. N.I. Vázquez-Fernández, T. Nyyssönen, M. Isakov, M. Hokka, V.-T. Kuokkala, Acta Mat. 176, 134-144 (2019)

14. M. Lindroos, A. Laukkanen, M. Isakov, T. Andersson, T. Pinomaa, S. Forest, Crystal plasticity modeling of transformation plasticity effects in metastable austenitic stainless steel microstructures: From Plasticity to damage, and towards fracture, International Conference on Plasticity, Damage, and Fracture 2019, Jan. 3-9, Panama City, Panama (2019) 